\title{
FEDORENKO FINITE SUPERELEMENT METHOD AS SPECIAL GALERKIN APPROXIMATION ${ }^{1}$
}

\author{
M. GALANIN and E. SAVENKOV \\ Keldysh Institute of Applied Mathematics RAS \\ Russia, Moscow, 125047, Miusskay square, 4 \\ E-mail: galan@keldysh.ru
}

Received September 29, 2001; revised December 11, 2001

\begin{abstract}
In this work we introduce variational equation which natural Petrov-Galerkin approximation leads to Fedorenko Finite Superelement Method (FSEM). FSEM is considered as Petrov-Galerkin approximation of the certain problem for traces of boundary-value problem solution at the boundaries of some subdomains (superelements). Iterative methods of solution of the same problem are well known domain decomposition methods. Some numerical results are presented.
\end{abstract}

\section{INTRODUCTION}

This paper considers some results on error analysis and applications of Fedorenko Finite Superelement Method [2; 10; 11; 12]. Error analysis for one variant of this method is presented in $[7 ; 8]$. Using the variational equation constructed below and suitable Petrov-Galerkin approximation one can consider a whole class of FSEM methods, not only the method introduced in original works of Fedorenko and his colleagues. In this paper we consider FSEM for Poisson equation in multiply-connected two-dimensional domain, but all results can be generalized over equations with arbitrary positively defined divergent second order elliptic operators.

FSEM belongs to the class of methods which reduce the initial boundary value problem in whole domain to a number of boundary value problems in

\footnotetext{
${ }^{1}$ This work was partially supported by Russian Fund for Basic Researches (projects 00-01-00169, 01-01-06265).
} 
subdomains. Such methods (for example, domain decomposition methods) are widely used and studied currently. They are naturally parallelizable and can be effectively realized on computers with parallel or multiprocessor architecture. The theoretical background for this methods is Poincare-Steklov operator's theory, which was introduced firstly in works of V. Agoshkov and V. Lebedev as effective tool for analysis of domain-decomposition methods (see review in [4]). This work also widely uses this formalism.

\section{PROBLEM FORMULATION}

We shall use conventional notations for functional spaces of smooth functions and functions with distributional derivatives $[1 ; 4 ; 6]$.

Let us consider the following Dirichlet boundary value problem for Poisson equation in domain $\Omega \subset \mathbb{R}^{2}$. Find $u \in W_{2}^{1}(\Omega,-\Delta)$ such that

$$
\begin{array}{r}
-\Delta u=f, \quad x \in \Omega, \\
\left.u\right|_{\partial \Omega}=g,
\end{array}
$$

where $f \in L_{2}(\Omega), \quad g \in W_{2}^{1 / 2}(\partial \Omega)$,

$$
W_{2}^{1}(\Omega,-\Delta)=\left\{u: u \in W_{2}^{1}(\Omega),-\Delta u \in L_{2}(\Omega)\right\} .
$$

Here we suppose that $\Omega \subset \mathbb{R}^{2}$ is an open multiply-connected domain which can be generated from open connected domain $\Omega_{0} \subset \mathbb{R}^{2}$ by elimination of some disjoint circles $\left\{\bar{S}_{i}\right\}$ ("wells") $\underset{i}{\cup} \bar{S}_{i} \subset \Omega_{0}, \Omega=\Omega_{0} \backslash \cup \bar{S}_{i=1}$. We denote by $\Gamma_{0}=\partial \bar{\Omega}_{0}, \Gamma_{i}=\partial \bar{S}_{i}$ the exterior and inner boundary of $\Omega$, respectively. Therefore, we have $\bar{\Omega}=\Omega \cup \Gamma, \Gamma \equiv \partial \Omega=\Gamma_{0} \cup\left(\cup_{i} \partial \bar{S}_{i}\right)$. Boundary condition (2.2) defines the trace of the solution at the whole disconnected boundary of $\Omega$.

\section{WEAK FORMULATIONS}

\subsection{Green's formula}

Green's formula is the main tool for construction and analysis of weak formulations of boundary-value problems. We will use the following variant of Green's formula in $W_{2}^{1}(\Omega)$, which follows from abstract Green's formula [6, p.188]. It states that there exists the unequally defined operator

$$
\delta: W_{2}^{1}(\Omega,-\Delta) \rightarrow W_{2}^{-1 / 2}(\partial \Omega),
$$

such that $a(u, v)=(-\Delta u, v)_{\Omega}+\langle\delta u, \gamma v\rangle_{\partial \Omega}, \forall u \in W_{2}^{1}(\Omega,-\Delta), v \in W_{2}^{1}(\Omega)$, $W_{2}^{1}(\Omega,-\Delta)=\left\{u \in W_{2}^{1}(\Omega):-\Delta u \in L_{2}(\Omega)\right\}, a(u, v)=\int_{\Omega} \nabla u \cdot \nabla v d \Omega$. Here 
$\gamma: W_{2}^{1}(\Omega) \rightarrow W_{2}^{1 / 2}(\partial \Omega)$ is a trace operator. If $u \in W_{2}^{2}(\Omega)$ and boundary $\partial \Omega$ is sufficiently smooth, then

$$
\delta u=\frac{\partial u}{\partial \vec{n}}, \quad\langle\delta u, \gamma v\rangle_{\partial \Omega}=\int_{\partial \Omega} \frac{\partial u}{\partial \vec{n}} v d \gamma .
$$

\subsection{Classical weak equation}

Classical weak formulation (2.1)-(2.2) is given by [6]. Find $u \in W_{2}^{1}(\Omega)$ such that

$$
\begin{aligned}
\left.u\right|_{\partial \Omega}=g, \quad g \in W_{2}^{1 / 2}(\partial \Omega), \\
\stackrel{\circ}{1}(\Omega) ; \quad f \in L_{2}(\Omega) .
\end{aligned}
$$

Problems (2.1)-(2.2) and (3.1)-(3.2) are equivalent [6, chapter 6]. Formulation (3.1) - (3.2) is also valid if $f \in W_{2}^{-1}(\Omega)$, but in this case it is not equivalent to $(2.1)-(2.2)$.

\section{SUPERELEMENTS}

Suppose that domain $\bar{\Omega}_{0}$ is decomposed in $K$ subdomains $\left\{\bar{\Omega}_{0, k}\right\}_{k=1}^{K}: \bar{\Omega}_{0}=$ $\bigcup_{k=1}^{K} \bar{\Omega}_{0, k}, \quad \bar{\Omega}_{0, k}=\Omega_{0, k} \cup \Gamma_{0, k}, \quad \Gamma_{0, k}=\partial \bar{\Omega}_{0, k}$, in such a way, that every $\bar{S}_{i}$ is an inner subdomain of $\Omega_{0, k}$ and every $\Omega_{0, k}$ contains no more then one "well" $S_{i}$ and decomposition is regular. Let $\Omega_{k}=\Omega \cap \Omega_{0, k}$. Then $\bar{\Omega}=\bigcup_{k=1}^{K} \bar{\Omega}_{k}$. We can represent boundary $\partial \Omega_{k}$ of domain $\Omega_{k}$ as $\partial \Omega_{k}=\gamma_{0, k} \cup \gamma_{k}, \gamma_{0, k}=\partial \Omega \cap \partial \Omega_{k}$, $\gamma_{k}=\partial \Omega_{k} \backslash \gamma_{0, k}$. We shall say that $\Omega_{k}$ are superelements. Since all subdomains are polygons, they have outer normals almost everywhere.

\section{VARIATIONAL EQUATION IN THE TRACE SPACES}

\subsection{Poincare-Steklov operator}

Let the operator $G: W_{2}^{1 / 2}(\partial \Omega) \rightarrow W_{2}^{1}(\Omega)$ is such that

$$
\begin{array}{r}
G \varphi=u, \\
\left.u\right|_{\partial \Omega}=\varphi, \quad \varphi \in W_{2}^{1 / 2}(\partial \Omega), \\
a(u, v)=0, \quad \forall v \in \stackrel{\circ}{2}_{2}^{1}(\Omega) .
\end{array}
$$

It is well-known that $G$ is a linear continuous operator, e.g. $\forall c_{1}, c_{2} \in \mathbb{R}$

$$
G\left(c_{1} \varphi_{1}+c_{2} \varphi_{2}\right)=c_{1} G\left(\varphi_{1}\right)+c_{2} G\left(\varphi_{2}\right) ; \quad\|G \varphi\|_{W_{2}^{1}(\Omega)} \leq C\|\varphi\|_{W_{2}^{1 / 2}(\partial \Omega)},
$$


where $C>0$ is independent from $\varphi$.

We define the following Poincare-Steklov operator:

$P: W_{2}^{1 / 2}(\partial \Omega) \rightarrow W_{2}^{-1 / 2}(\partial \Omega), P \varphi=\delta G \varphi$. If solution $u=G \varphi \in W_{2}^{2}(\Omega)$, then $P \varphi=\partial G \varphi / \partial \vec{n}$. It follows from Green's formula that $\forall \varphi_{1}, \varphi_{2} \in W_{2}^{1 / 2}(\partial \Omega)$ : $\left\langle P \varphi_{1}, \varphi_{2}\right\rangle=\left\langle\varphi_{1}, P \varphi_{2}\right\rangle$. We will denote operators $G$ and $P$ corresponding to domain $\Omega_{k}$ as $G_{k}$ and $P_{k}$.

\subsection{Variational equation}

Let's consider the following Hilbert space $\tilde{H}$ :

$$
\tilde{H}=\prod_{k=1}^{K} L_{2}\left(\partial \Omega_{k}\right), \quad\langle\mu, \nu\rangle_{\tilde{H}}=\sum_{k=1}^{K}\left\langle\mu_{k}, \nu_{k}\right\rangle_{\partial \Omega_{k}}, \quad\|\mu\|_{\tilde{H}}=\sqrt{\langle\mu, \mu\rangle_{\tilde{H}}} .
$$

Let $H$ be a subspace of $\tilde{H}$ :

$$
\begin{gathered}
H=\left\{\mu \in \tilde{H}: \quad \forall i, j \in\{1, \ldots, K\} \text { such that } \gamma_{i j}=\partial \Omega_{i} \cap \partial \Omega_{j} \neq \emptyset\right. \\
\left.\left.\mu_{i}\right|_{\gamma_{i j}}=\left.\mu_{j}\right|_{\gamma_{i j}} \text { almost everywhere }\right\}
\end{gathered}
$$

Also we consider the Hilbert space

$$
\tilde{X}=\prod_{k=1}^{K} W_{2}^{1 / 2}\left(\partial \Omega_{k}\right), \quad\|\mu\|_{\tilde{X}}=\sum_{k=1}^{K}\left\|\mu_{k}\right\|_{W_{2}^{1 / 2}\left(\partial \Omega_{k}\right)}
$$

and it's subspaces

$$
\begin{aligned}
X & =\left\{\mu \in \tilde{X}: \exists v \in W_{2}^{1}(\Omega), \mu_{k}=\left.v\right|_{\partial \Omega_{k}}\right\}, \\
X_{0} & =\left\{\mu \in \tilde{X}: \exists v \in \underset{W_{2}^{1}}{\left.W^{\prime}(\Omega), \mu_{k}=\left.v\right|_{\partial \Omega_{k}}\right\} .}\right.
\end{aligned}
$$

Let $\tilde{X}^{\prime}=\prod_{k=1}^{K} W_{2}^{-1 / 2}\left(\partial \Omega_{k}\right)$ be a dual space to $\tilde{X}$. Since $W_{2}^{1 / 2}\left(\partial \Omega_{k}\right) \subset$ $L_{2}\left(\partial \Omega_{k}\right) \subset W_{2}^{-1 / 2}\left(\partial \Omega_{k}\right)$, we have $\tilde{X} \subset H \subset \tilde{X}^{\prime}$. Notice that $X^{\prime}=\tilde{X}^{\prime}$. In the spaces $W_{2}^{-1 / 2}\left(\partial \Omega_{k}\right)$ and $X^{\prime}$ we use standard dual norms. Now we consider Poincare-Steklov operators $P_{k}$ and a bilinear form

$$
b(\mu, \nu)=\sum_{k=1}^{K}\left\langle P_{k} \mu_{k}, \nu_{k}\right\rangle_{\partial \Omega_{k}}
$$

defined for all $\mu, \nu \in \tilde{X}$. The following lemma is valid. 
Lemma 5.1. Form $b(\cdot, \cdot)$ is a continuous symmetric positively defined bilinear form in $X_{0} \times X_{0}$, e.g. $\forall \mu, \nu \in X_{0}: \quad|b(\mu, \nu)| \leq c_{2}\|\mu\|_{X_{0}}\|\nu\|_{X_{0}} ; \forall \mu \in X_{0}$ : $c_{1}\|\mu\|_{X_{0}}^{2} \leq b(\mu, \mu)$.

Now we can formulate the following problem: find $\varphi \in X_{0}$ such that

$$
b(\varphi, \psi)=F(\psi), \quad \forall \psi \in X_{0},
$$

where $F(\psi)$ is a continuous linear functional on $X, F \in X^{\prime}$.

It follows from Lax-Milgramm lemma that this problem has the unique solution. Finally, we can prove the following theorem.

Theorem 5.1. Let $F(\psi)=-b(\tilde{\varphi}, \psi), \psi \in X ; \tilde{\varphi}=\left\{\tilde{\varphi}_{k}\right\}_{k=1}^{K}=\left\{\left.v\right|_{\partial \Omega_{k}}\right\}_{k=1}^{K} \in$ $X, F \in X^{\prime}$, where $v=\left\{v_{k}\right\}_{k=1}^{K}$ is such a function that

$$
-\Delta v_{k}=f \quad \text { in } \quad \Omega_{k},\left.\quad v_{k}\right|_{\partial \Omega_{k}}=\left.w\right|_{\partial \Omega_{k}}, k=\overline{1, K},
$$

and $w \in W_{2}^{1}(\Omega),\left.w\right|_{\partial \Omega}=g$ is any function satisfying (2.2). Then solution $u=\left\{u_{k}\right\}_{k=1}^{K} \in W_{2}^{1}(\Omega)$ of the problem $(2.1)-(2.2)$ in superelement $\Omega_{k}$ is given by $u_{k}=G_{k} \varphi_{k}+v_{k}, k=\overline{1, K}$.

Suppose that in (5.3) the functional $F \in X^{\prime}=\tilde{X}^{\prime}$ belongs to $\tilde{H}=\tilde{H}^{\prime} \subset \tilde{X}^{\prime}$. Then $F(\psi)=\langle F, \psi\rangle=\sum_{k=1}^{K} \int_{\partial \Omega_{k}} F_{k} \psi_{k} d l$ and we can reformulate the problem (5.3) in the following way. Find $\varphi \in X_{0}$ such that

$$
b(\varphi, \psi)=F(\psi), \quad \forall \psi \in H_{0},
$$

where $H_{0}$ is subspace of $H$, which consists of functions, vanishing on $\gamma_{0, k}=$ $\partial \Omega_{k} \cap \partial \Omega$. In this case the bilinear form $b(\cdot, \cdot)$ is defined in $X_{0} \times H_{0}$ and it is not symmetric.

\section{APPROXIMATIONS}

Finite Superelement Method is a Petrov-Galerkin approximation of (5.4). Petrov-Galerkin approximations of abstract and boundary-value problems are considered in $[3 ; 5]$.

For the approximation of the problem (5.4) one should choose the finite dimensional subspace $X_{0, h}$ which is a span of function system $\left\{\varphi_{h}^{(i)}\right\}_{i=1}^{N} \subset X_{0}$, and the finite dimensional subspace $H_{0, h}$, which is a span of function system $\left\{\psi_{h}^{(i)}\right\}_{i=1}^{N} \subset H_{0}$. We find the approximate solution $\varphi_{h} \in X_{0, h}$ of the problem (5.4) as the following linear combination:

$$
\varphi_{h}=\sum_{i=1}^{N} a_{i} \varphi_{h}^{(i)}
$$


satisfying the conditions:

$$
b\left(\varphi_{h}, \psi_{h}\right)=F\left(\psi_{h}\right), \quad \forall \psi_{h} \in H_{0, h} .
$$

Choosing different subspaces $X_{0, h}$ and $H_{0, h}$ one can get different variants of FSEM. In all cases the algorithm implementation is given by:

1. For every function $\varphi_{h}^{(i)}$ compute and store the following functions:

$$
\begin{gathered}
u_{h}^{(i)}=G \varphi_{h}^{(i)}=\left\{G_{k} \varphi_{h, k}^{(i)}\right\}_{k=1}^{K}, \\
\Pi_{h}^{(i)}=P \varphi_{h}^{(i)}=\left\{P_{k} \varphi_{h, k}^{(i)}\right\}_{k=1}^{K}=\left\{\partial u_{h, k}^{(i)} / \partial n_{k}\right\}_{k=1}^{K} .
\end{gathered}
$$

To compute $u_{h}^{(i)}$ one should solve in each $\Omega_{k}$ the boundary value problem with the boundary condition, defined by function $\varphi_{h}^{(i)}$.

2. After solving problem (6.2), we get approximate solution $\varphi_{h}$ of the problem (5.4). Then the approximate solution of the boundary value problem (3.1)(3.2) is given by $u_{h}=\sum_{i=1}^{N} a_{i} u_{h}^{(i)}$.

We consider two variants of $X_{0, h}$ and $H_{0, h}$.

\subsection{Fedorenko FSEM}

On $\bigcup_{k=1}^{K} \partial \Omega_{k}$ we consider the set of nodes $M_{h}$ consisting of vertices of polygons $\Omega_{k}$. For every node $M_{i} \in M_{h}$ we define a basis function $\varphi_{h}^{(i)} \in X_{0, h}$ in the following way: $\varphi_{h}^{(i)}\left(M_{j}\right)=\delta_{i j}$. At exterior bounds of superelements $\varphi_{h}^{(i)}$ is a linear function, which vanishes at inner bounds ("wells"). Function $\psi_{h}^{(i)}$ is defined as characteristic function of $\omega_{i}$. If we choose $X_{0, h}$ and $H_{0, h}$ as shown above, we get the first order Fedorenko FSEM.

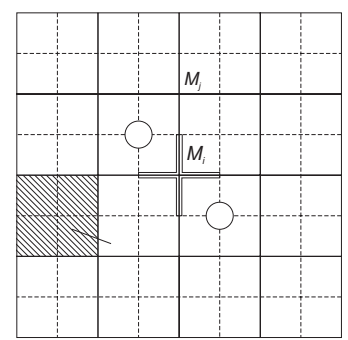

Figure 1. Domain and superelements.

\subsection{Bubnov-Galerkin FSEM}

Bubnov-Galerkin FSEM corresponds to the case when $\varphi_{h}^{(i)}=\psi_{h}^{(i)}$ (systems of test and trial functions are equal). Basis functions $\varphi_{h}^{(i)}$ are defined as presented above. 


\section{BUBNOV-GALERKIN FSEM ERROR ANALYSIS}

It is well known that the error analysis of Bubnov-Galerkin approximations leads to the analysis of the interpolation errors of the solution $\varphi$ by finite dimensional subspace $X_{0, h}$. According to Sea lemma [1, p.109] we obtain:

$$
\left\|\varphi-\varphi_{h}\right\|_{X} \leq M \inf _{\psi_{h} \in V_{h}}\left\|\varphi-\psi_{h}\right\|_{X} \leq\|\varphi-\tilde{\varphi}\|_{X}, \quad M=c_{2} / c_{1} .
$$

Here $\varphi_{h}$ is the solution of (6.2) with $\psi_{h}^{(i)}=\varphi_{h}^{(i)}$ and $\tilde{\varphi} \in X_{0, h}$ is the interpolating element of the exact solution $\varphi$ in $X_{0, h}$.

Now we can estimate $\|\varphi-\tilde{\varphi}\|_{X}$ in the case of polynomial interpolation. Additionally we suppose that $u \in W_{2}^{1}(\Omega) \cap C^{2}(\Omega)$. In this case $i$-th basis function $\varphi_{h}^{(i)} \in X_{0, h}$ is defined as interpolating polynomial of arbitrary function from $\varphi \in X_{0}$, which is equal to one in $i$-th node and is equal to zero in all other nodes.

Let $I=[a, b] \subset \mathbb{R}$. We consider $W_{2}^{1 / 2}(I)$ space on $I$ with norm

$\|\varphi\|_{W_{2}^{1 / 2}(I)}^{2}=\|\varphi\|_{L_{2}(I)}^{2}+\int_{I} \int_{I} \frac{|\varphi(x)-\varphi(y)|^{2}}{|x-y|^{2}} d x d y ;\|\varphi\|_{L_{2}(I)}^{2}=\int_{I} \varphi^{2}(x) d x$.

Let $\varphi \in C^{1}(I) \cap W_{2}^{1 / 2}(I)$. It follows from (7.1) that

$$
\|\varphi\|_{W_{2}^{1 / 2}(I)}^{2} \leq\|\varphi\|_{L_{2}(I)}^{2}+\alpha^{2}|I|^{2}, \quad \alpha=\max _{\xi \in I}\left|\varphi^{\prime}(\xi)\right|, \quad|I|=|b-a| .
$$

Suppose that $\varphi \in C_{0}^{1}(I)$. Then we obtain

$$
\|\varphi\|_{W_{2}^{1 / 2}(I)}^{2} \leq \frac{1}{3} \alpha^{2}|I|^{3}+\alpha^{2}|I|^{2}=\alpha^{2}|I|^{2}\left(\frac{1}{3}|I|+1\right) .
$$

Now we consider the domain $\Omega \subset \mathbb{R}^{2}$ and its regular decomposition in $K$ polygons $\Omega_{k}, k=\overline{1, K}$. Let $\Omega_{0}=\mathbb{R}^{2} \backslash \Omega$. Let $\gamma_{i, j}$ be a common edge of subdomains $\Omega_{i}$ and $\Omega_{j}, i, j=\overline{0, K}, i<j$. We denote the set of all such pairs by $J$. Functional space $X$ can be defined as follows:

$$
X=\left\{\mu=\left\{\mu_{k}\right\}_{k=1}^{K} \in \tilde{X}:\left.\quad \forall(i, j) \in J \quad \mu_{i}\right|_{\gamma_{i, j}}=\left.\mu_{j}\right|_{\gamma_{i, j}}\right\} .
$$

We shall denote $\left\|\mu_{k}\right\|_{W_{2}^{1 / 2}\left(\partial \Omega_{k}\right)}^{2}=\sum_{i, j}\left\|\mu_{k}\right\|_{W_{2}^{1 / 2}\left(\gamma_{i, j}\right)}^{2}$, where summation is done through all pairs $(i, j) \in J$, such that $\gamma_{i, j} \in \partial \Omega_{k}$. Then we obtain

$$
\|\mu\|_{X}^{2}=\sum_{k=1}^{K}\left\|\mu_{k}\right\|_{W_{2}^{1 / 2}\left(\partial \Omega_{k}\right)}^{2}=2 \sum_{i, j \in J}\left\|\mu_{k}\right\|_{W_{2}^{1 / 2}\left(\gamma_{i, j}\right)}^{2} .
$$


At the $\bigcup_{k=1}^{K} \partial \Omega_{k}$ we consider the set of nodes $M_{h}$. We assume that every edge $\gamma_{i, j}$ has the same number of nodes $L+1 \geq 2$ and two of this nodes always correspond to the vertices of $\gamma_{i, j}$. Interpolating element of $\varphi \in X$ at every edge $\gamma_{i, j}$ is defined as ordinary interpolating polynom of $\left.\varphi\right|_{\gamma_{i, j}}$ over $L+1$ nodes at $\gamma_{i, j}$. Here we assume that $\varphi_{k} \in W_{2}^{1 / 2}\left(\partial \Omega_{k}\right)$ is continuous function and $\left.\varphi\right|_{\gamma_{i, j}} \in C^{1}\left(\gamma_{i, j}\right)$. Combining (7.3) and (7.2), we reduce the estimates for $\|\varphi-\tilde{\varphi}\|_{X}$ to the error estimates of ordinary polynomial interpolation at $\gamma_{i, j}$.

Let $M_{i, j}$ be a set of nodes which belong to edge $\gamma_{i, j}$. At edge $\gamma_{i, j}$ we consider some coordinate system $O s$ with the origin at one of the vertices of $\gamma_{i, j}$. Let $\left\{0=s_{0}, s_{1}, \ldots, s_{L-1}, s_{L}=\left|\gamma_{i, j}\right|\right\}$ be coordinates of nodes from $M_{i, j}$. By $\varphi_{i, j}$ and $\tilde{\varphi}_{i, j}$ we denote the restrictions of $\varphi$ and $\tilde{\varphi}$ on $\gamma_{i, j}$. Then interpolation errors obey the following inequalities [9]

$$
\begin{aligned}
\max _{s \in \gamma_{i, j}}\left|\varphi_{i, j}-\tilde{\varphi}_{i, j}\right| & \leq \frac{\alpha_{L+1, i, j}}{(L+1) !}\left|\gamma_{i, j}\right|^{L+1}, \\
\max _{s \in \gamma_{i, j}}\left|\varphi_{i, j}^{\prime}-\tilde{\varphi}_{i, j}^{\prime}\right| \leq \frac{\alpha_{L+1, i, j}}{L !}\left|\gamma_{i, j}\right|^{L} ; \quad \alpha_{L+1, i, j} & =\max _{s \in \gamma_{i, j}}\left|\varphi^{(L+1)}(s)\right| .
\end{aligned}
$$

Finally, combining (7.2), (7.3) and (7.4) we obtain

$$
\|\varphi-\tilde{\varphi}\|_{X}^{2} \leq 2 \sum_{i, j \in J} \frac{\alpha_{L+1, i, j}^{2}}{(L !)^{2}}\left|\gamma_{i, j}\right|^{2(L+1)}\left(\frac{1}{3}\left|\gamma_{i, j}\right|+1\right) .
$$

Here $\alpha_{L+1, i, j}$ depend on the domain $\Omega$ decomposition.

From the well known properties of polynomial interpolation it follows that most interesting cases are $L=1$ and $L=2$. It also follows that the traces of the solution at the boundaries of superelements should be sufficiently smooth functions. But inside superelements the solution can have singularities or large gradients. Suitable way of interpolation also depends on superelement geometry, type and location of singularities. In general case at every edge $\gamma_{i, j}$ one can use different kind of interpolation. Taking into account the continuity of operators $G_{k}$ we obtain that the convergence of $\varphi_{h}$ to $\varphi$ in $X$ leads to the convergence of $u_{h}$ to $u$ in $W_{2}^{1}(\Omega)$.

It is interesting to study the dependence of error estimates on distance between "well" and superelement boundary. We consider the boundary value problem for the Laplace equation in the square domain with one "well" in the center of the square. We assume that edges of the square have length $l$ and are parallel to the coordinate axes, the "well" has radius $r_{c}$ and it is situated at the origin of coordinates. We will use the following function as an exact solution of the model problem:

$$
u(r, \varphi)=u_{c}+\ln \left(\frac{r_{c}}{r}\right),
$$

where $(r, \varphi)$ are polar coordinates in $O x y$. This function satisfies the Laplace equation in $\Omega$ and it is equal to $u_{c}$ at the boundary of the "well". 

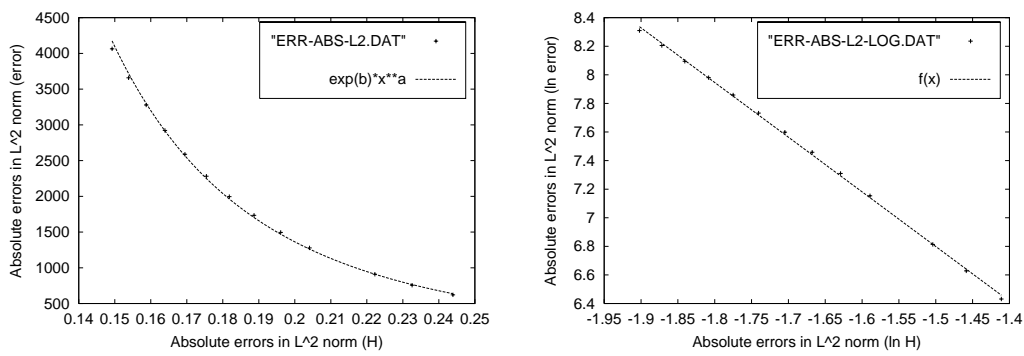

Figure 2. Errors of the solution in $H^{2}$.

We decompose domain $\Omega$ into superelements with lines which are parallel to the coordinate axes. Let distance between this lines is equal to $H=l /(2 N+1)$, $N \geq 1$. So every superelement is a square with length of edge equal to $H$. The "well" is situated in the central superelement. Total number of superelements is equal to $K=(2 N+1)^{2}$. We will use the Bubnov-Galerkin approximations to solve this problem.

Since we know the exact solution (7.6) and error estimate (7.5), we can obtain error estimates as a function of $H$. Estimates (7.5) in this case $(L=1$, $\left.\gamma_{i, j}=H\right)$ give

$$
\left\|\varphi-\varphi_{h}\right\|_{X}^{2} \leq 4 l^{2} H^{2}\left(\frac{1}{3} H+1\right) \max _{\gamma_{i, j}} \alpha_{2, \gamma_{i, j}}^{2} .
$$

Taking into account that edges $\gamma_{i, j}$ are parallel to coordinate axes, using the formula for the exact solution (7.6) and symmetry of the domain, we obtain

$$
\max _{\gamma_{i, j}} \alpha_{2, \gamma_{i, j}}=\max _{\gamma_{i, j}} \max _{s \in \gamma_{i, j}}\left|\varphi^{(2)}(s)\right| \leq\left.\max _{x \in[-l / 2 ;+l / 2]}\left|\frac{\partial^{2} u(x, y)}{\partial x^{2}}\right|\right|_{y=H / 2}=\frac{4}{H^{2}}
$$

Finally, we have: $\left\|\varphi-\varphi_{h}\right\|_{X}^{2} \leq 64 \frac{l^{2}}{H^{2}}\left(\frac{1}{3} H+1\right)$. Thus it follows that error is decreasing when distance between "well" and superelement boundary is increasing.

Analysis of numerical tests show (see Fig.2) $\left\|\varphi-\varphi_{h}\right\|_{H}^{2} \sim \frac{1}{H^{1.9}}$.

The obtained strong error dependence on the distance between "well" and superelement boundary proves the importance of a priori analysis of the initial boundary value problem and its singularities.

\section{REFERENCES}

[1] F. Ciarlet. Finite element method for elliptic problems. Moscow, Mir, 1980. (in Russian)

[2] R.P. Fedorenko. Introduction into computational physics. Moscow, MFTI Publishing, 1994. (in Russian) 
[3] M.A. Krasnoselky, G.M. Vainicco, P.P. Zabreiko and Y.B. Rutitsky. Approximate solution of operator equations. Moscow, Nauka, 1969. (in Russian)

[4] G.I. Marchuk. Methods of computational mathematics. Moscow, Nauka, 1989. (in Russian)

[5] G.I. Marchuk and V.I. Agoshkov. Introduction in mesh-projection methods. Moscow, Nauka, 1981. (in Russian)

[6] J.-P. Oben. Approximate solution of elliptic problems. Moscow, Mir, 1977. (in Russian)

[7] V.V. Repiah. Application of one variant of finite superelemnts method in elasticity problems. J. Comp. Math. and Math. Phys., 24(11), 1643 - 1653, 1986. (in Russian)

[8] V.V. Repiah. Error analysis of approximate finite superelements method. J. Comp. Math. and Math. Phys., 30(7), 963 - 983, 1989. (in Russian)

[9] V.S. Ryabenky. Introduction into computational mathematics. Moscow, Nauka, 1994. (in Russian)

[10] L.G. Strakhovskaja and R.P. Fedorenko. On one variant of finite elements method. $J$. of Comp. Math. and Math. Phys., 19(4), 950-960, 1979.

[11] L.G. Strakhovskaja and R.P. Fedorenko. The calculation of diffusion in multi-connected region by finite superelements method. Preprint KIAM AS USSR, 171, 1987. (in Russian)

[12] L.G. Strakhovskaja and R.P. Fedorenko. The calculation of the stress state of $3 d$ composite body by finite superelements method. Preprint KIAM AS USSR, 67, 1994. (in Russian)

\section{Fedorenko baigtinių elementų metodas kaip Galerkino metodo aproksimacija}

M. Galanin, E. Savenkov

Pateikta vienos variacinès lygties Petrovo-Galerkino aproksimacija, kuri veda i Fedorenko baigtinių elementų metodą. Jis nagrinejjamas kaip tam tikro uždavinio Petrovo-Galerkino aproksimacija kraštinių uždavinių sprendinių pėdsakams kai kurių sričių (superelementų) kraštuose. Tokių uždavinių iteraciniai sprendimo metodai yra gerai žinomi kaip srities dekompozicijos metodai. Pateikti skaitinių eksperimentų rezultatai. 